\title{
Relatos de profissionais sobre intercorrências em tratamentos estéticos corporais
}

\author{
Professionals' reports on intercurrences in aesthetic body treatments \\ Informes de los profesionales sobre las complicaciones en tratamientos estéticos corporales
}

Recebido: 18/11/2021 | Revisado: 24/11/2021 | Aceito: 26/11/2021 | Publicado: 09/12/2021

\author{
Thais Noable França Ribas \\ ORCID: https://orcid.org/0000-0002-7500-4191 \\ Faculdade Independente do Nordeste, Brasil \\ E-mail: thaisnoable@gmail.com \\ Juliana Braga Facchinetti Moura \\ ORCID: https://orcid.org/0000-0001-9621-801X \\ Faculdade Independente do Nordeste, Brasil \\ E-mail: julianafacchinetti@hotmail.com
}

\begin{abstract}
Resumo
A busca por tratamentos estéticos vem aumentando consideravelmente nos últimos tempos independentemente da idade e do sexo, objetiva-se o corpo perfeito, sem estrias, sem celulite e sem gordura localizada. O objetivo do estudo foi identificar quais são as intercorrências nos tratamentos estéticos corporais. O estudo se caracteriza como descritivo com abordagem quantitativa. Devido à situação do COVID -19, o estudo foi realizado de forma não presencial, através de um questionário no Google Forms. A pesquisa foi realizada com 28 profissionais da área da saúde como esteticistas, fisioterapeutas, enfermeiros e biomédicos atuantes na área da estética corporal. Foi possível identificar as intercorrências em tratamentos estéticos corporais que vão desde as mais simples as mais complexas como queimaduras, manchas e hiperplasia paradoxal. Observou-se também os recursos terapêuticos que mais apresentaram intercorrências e as principais condutas que os profissionais mantiveram diante das intercorrências. O tema é muito importante na atualidade, porém pouco se encontra publicações, que é de extrema relevância para os profissionais da saúde que atuam com estética corporal.
\end{abstract}

Palavras-chave: Estética; Efeitos adversos; Saúde.

\begin{abstract}
The search for aesthetic treatments has increased considerably in recent times regardless of age and gender, the objective is the perfect body, without stretch marks, cellulite, and localized fat. The objective of this study was to identify what are the complications of aesthetic body treatments. The study is characterized as descriptive with a quantitative approach. Due to the situation of COVID -19, the study was conducted in a non face-to-face manner, through a questionnaire on Google Forms. The research was carried out with 28 health professionals such as aestheticians, physical therapists, nurses and biomedical professionals working in the field of body aesthetics. It was possible to identify the complications in body aesthetic treatments that range from the simplest to the most complex such as burns, stains and paradoxical hyperplasia. It was also observed the therapeutic resources that presented the most complications and the main behaviors that the professionals had when facing these complications. The theme is very important nowadays, but few publications can be found, which is extremely relevant for health professionals who work with body esthetics.
\end{abstract}

Keywords: Aesthetics; Adverse effects; Health.

\begin{abstract}
Resumen
La búsqueda de tratamientos estéticos ha aumentado considerablemente en los últimos tiempos, independientemente de la edad y el sexo, el objetivo es el cuerpo perfecto, sin estrías, sin celulitis y sin grasa localizada. El objetivo del estudio fue identificar cuáles son las intercurrencias en los tratamientos estéticos corporales. El estudio se caracteriza por ser descriptivo con un enfoque cuantitativo. Debido a la situación del COVID -19, el estudio se realizó de forma no presencial, a través de un cuestionario en Google Forms. La investigación se llevó a cabo con 28 profesionales de la salud como esteticistas, fisioterapeutas, enfermeras y biomédicos que trabajan en el ámbito de la estética corporal. Se han podido identificar las complicaciones en los tratamientos de estética corporal, desde las más sencillas hasta las más complejas, como las quemaduras, las manchas y la hiperplasia paradójica. También se observaron los recursos terapéuticos que presentaron más complicaciones y las principales conductas que los profesionales mantuvieron ante las complicaciones. El tema es muy importante en la actualidad, aunque se encuentran pocas publicaciones, pero es de extrema relevancia para los profesionales de la salud que trabajan con la estética corporal.
\end{abstract}

Palabras clave: Estética; Efectos adversos; Salud. 


\section{Introdução}

A preocupação com o corpo ganhou muita importância na sociedade já que a beleza se reflete na autoestima e na qualidade de vida (Bastos \& Nogueira, 2020). A busca por tratamentos estéticos vem aumentando consideravelmente nos últimos tempos independentemente da idade e do sexo, objetiva-se o corpo perfeito, sem estrias, sem celulite e sem gordura localizada (Milani, 2020).

De acordo com Filgueiras (2018) além das mulheres serem o público que tradicionalmente mais procuram por serviços como estes, os homens estão se tornando cada vez mais vaidosos e buscando também estes serviços que até então era exclusividade do público feminino. As disfunções estéticas corporais que obtêm maior procura nas clínicas de estética são adiposidade localizada, celulite, flacidez e estrias.

Dentre as disfunções estéticas, a adiposidade localizada está entre as disfunções que mais acometem os indivíduos ao redor do mundo, e está associada a uma soma de hábitos que podem ser prejudiciais à saúde (Brito, et al., 2019). O tecido adiposo é um tipo especial de tecido conjuntivo onde se observa o predomínio de células adiposas, os adipócitos. O excesso de gordura é constituído por fatores genéticos, má alimentação, sedentarismo, metabolismo e desordens hormonais (Feller et al., 2018).

O fibroedemageloide (FEG), conhecido popularmente como celulite, é um distúrbio do panículo adiposo que afeta cerca de 80 a $90 \%$ das mulheres após a puberdade e atinge especialmente a região glútea e as coxas. Nesse sentido, o FEG se caracteriza por uma alteração circulatória, que causa edema local e compromete a microcirculação (Oliveira, et al., 2014).

A flacidez cutânea está associada basicamente a perda gradativa de elementos importantes da constituição do tecido conjuntivo como a elastina, os fibroblastos e por consequência colágeno, o que resulta na diminuição da firmeza entre as células, "afrouxando" a pele, ou, ainda, tracionando a estrutura acima da sua capacidade resistiva (Tassinary et al., 2018).

As estrias representam um problema bastante comum na população, principalmente em mulheres adolescentes, representadas por uma lesão dérmica por estiramento excessivo e progressivo do sistema tegumentar. De acordo com Queiroz et al. (2021) as estrias podem ser definidas como uma atrofia adquirida, e estão localizadas na camada dérmica, em consequência do estiramento das fibras de colágeno e elastina.

Tassinary et al. (2018) trazem que os recursos da eletroterapia como, os tipos de ultrassom sejam eles de: alta potência, focalizado, convencional, ultracavitação e a terapia combinada. Além de ondas de choque, criolipólise, radiofrequência, eletrolipoforese, corrente russa, a carboxiterapia, microgalvanopuntura, microdermoabrasão, microagulhamento, laser de baixa frequência, plataforma vibratória e endermologia são os procedimentos mais utilizados para o tratamento das afecções corporais.

Além da escolha adequada e conhecimento dos recursos terapêuticos, se atentar a biossegurança é fundamental, ela deve fazer parte da formação do profissional que atua na área da beleza e estética; sendo esse um diferencial para a classe desses profissionais, pois com isso, suas atividades são asseguradas a prática correta, reduzindo assim os riscos (Borges \& Scorza, 2016). A segurança nos procedimentos estéticos é algo que deve ser levado a sério, pois cuidar da beleza com segurança irá proporcionar a satisfação nos resultados e preservação da saúde (Carlesi, 2017 apud Trindade, et al., 2020).

Cada procedimento ou recursos terapêuticos apresentam indicações, contraindicações e podem gerar reações adversas e intercorrências. Dessa forma, este estudo tem o objetivo de identificar quais são as intercorrências de tratamentos estéticos corporais relatados por profissionais no tratamento da gordura localizada, fibroedemageloide, estrias e flacidez cutânea. Sendo assim, os profissionais que atuam com a estética poderão utilizar dessas informações para minimizar a quantidade de intercorrências ao realizar estes procedimentos. 


\section{Metodologia}

O presente estudo se caracteriza como descritivo com abordagem quantitativa. Devido à situação do COVID -19, o estudo foi realizado de forma não presencial, por meio do ambiente virtual através de um questionário elaborado pelo Google Forms, sendo aplicado através de convites virtuais e individuais, para cada profissional.

A pesquisa foi iniciada somente após o projeto ser aprovado pelo Comitê de Ética e Pesquisa da Faculdade Independente do Nordeste (CEP/FAINOR), sob parecer de aprovação $\mathrm{N}^{\circ}$ 4.815.910. Foram respeitados todos os princípios éticos das resoluções n 466 de 2012 e n 510 de 2016 do Conselho Nacional de Saúde (CNS), em que estão descritos todas as diretrizes, normas e regulamentos que envolvem pesquisa com seres humanos.

A pesquisa foi realizada com 28 profissionais da área da saúde como: esteticistas com graduação em curso tecnólogo em estética, fisioterapeutas, enfermeiros e biomédicos atuantes na área da estética. Foram incluídos os profissionais da saúde da cidade de Vitoria da Conquista - Bahia que atuavam com estética; e foram excluídos aqueles profissionais que não atuavam com disfunções corporais.

A busca por profissionais que atuavam com estética na cidade de Vitória da Conquista - Bahia. foi realizada através de pesquisa nas redes sociais (Instagram, Facebook e Whatsapp). Após identificado os contatos destes profissionais da estética, os pesquisadores fizeram o convite de forma individual, através de caixa de mensagem, e-mail ou whatsapp de cada profissional. No convite tinham informações do projeto da pesquisa, como os objetivos, riscos e benefícios do estudo. Em seguida, após os convidados terem aceitado participar, foi enviado pela mesma plataforma, um documento com o Termo de Consentimento Livre e Esclarecido (TCLE) para que registrassem a concordância em participar da pesquisa. O questionário desenvolvido pelas pesquisadoras também foi disponibilizado de forma online, através do e-mail: tccintercorrenciascorporais@gmail.com, com link na plataforma Google Forms, para o preenchimento.

No questionário havia questões sobre os aspectos sociodemográficos e perfil ocupacional como: idade, cidade de atuação, profissão, tempo de formação, especialização, tempo de atuação na área da estética corporal. Sequencialmente foi aplicado o questionário com perguntas relacionadas sobre quais tratamentos corporais já provocaram intercorrências durante o tempo de prática profissional, quais intercorrências foram identificadas em cada tratamento e quais foram às condutas profissionais antes e após o surgimento das intercorrências.

Depois de concluída a coleta de dados, foi feito o download de todos os dados coletados para um dispositivo eletrônico local, apagado todos os registros feitos na plataforma Google Forms. A partir dos dados coletados com os profissionais, foi realizada a análise dos resultados. Os dados quantitativos foram encaminhados e descritos através do Microsoft Excel para forma de tabelas, para melhor compreensão dos resultados, e consequentemente foi feito uma análise descritiva das variáveis do estudo.

\section{Resultados e Discussão}

Os resultados dos dados coletados serão expostos nesse tópico e exibidos em forma de tabelas e comentários, a fim de que se tenha uma melhor compreensão dos resultados obtidos. As perguntas foram elaboradas e descritas de acordo com o objetivo da pesquisa.

A pesquisa foi feita com 28 participantes, com idade entre 24 a 46 anos de idade, sendo $96,4 \%$ do sexo feminino. A maioria dos profissionais eram esteticista $(46,4 \%)$ ou fisioterapeutas $(32,1 \%)$. Cerca de $85,7 \%$ desses profissionais afirmaram que possuíam especialização na área da estética corporal, e 46,4\% relataram ter entre 5 a 10 anos de atuação na área. (Tabela 1) 
Tabela 1. Questionário Sociodemográfico e perfil ocupacional. Vitória da Conquista, 2021.

\begin{tabular}{|l|l|}
\hline Idade & \\
\hline & 24 a 46 anos \\
\hline Sexo & \\
\hline Feminino & $96,4 \%$ \\
\hline Masculino & $3,6 \%$ \\
\hline Profissão & \\
\hline Esteticista & $46,4 \%$ \\
\hline Fisioterapeuta & $32,1 \%$ \\
\hline Biomédico(a) & $10,2 \%$ \\
\hline Enfermeiro(a) & $10,2 \%$ \\
\hline Especialização em estética corporal & \\
\hline Sim & $85,7 \%$ \\
\hline Não & $14,7 \%$ \\
\hline Tempo de atuação com estética corporal & \\
\hline Menos de 5 anos & $35,7 \%$ \\
\hline Entre 5 a 10 anos & $46,4 \%$ \\
\hline Mais de 10 anos & $17,9 \%$ \\
\hline
\end{tabular}

Fonte: Autores (2021).

Após traçar o perfil sociodemográfico e ocupacional destes profissionais, eles foram questionados sobre intercorrências e 89,3\% dos profissionais afirmaram que não tiveram intercorrências nos tratamentos estéticos corporais.

Existem diversos tratamentos estéticos corporais, dentre eles, o ultrassom convencional que pode promover efeitos mecânicos e térmicos sendo indicado para tratar diversas disfunções estéticas, como a adiposidade localizada e fibroedemagelóide. A terapia ultrassônica pode parecer simples, mas requer uma atenção, pois caso seja utilizada de forma inadequada pode causar queimaduras e microlesões no tecido (Borges \& Scorza, 2016). Entretanto, apesar da literatura falar sobre as possíveis intercorrências, todos os profissionais entrevistados afirmaram que nunca tiveram intercorrências com o uso do ultrassom.

Conforme Pereira (2019) diz, a radiofrequência é um aparelho que é capaz de gerar um calor profundo no interior dos tecidos, aquecendo de dentro para fora. De acordo com Silva et al. (2017), por ser uma técnica que provoca um aquecimento intenso nos tecidos pode surgir edemas, hiperpigmentação pós- inflamatória, pápulas eritematosas, bolhas e queimaduras superficiais. No presente estudo, apenas $7,1 \%$ dos profissionais relataram intercorrências com o uso da radiofrequência, manifestando petéquias e queimaduras após a utilização deste recurso eletroterapêutico.

A criolipólise é um recurso que provoca um resfriamento não invasivo no tecido adiposo, com temperaturas baixas entre $-5^{\circ} \mathrm{C}$ até $-15^{\circ} \mathrm{C}$, causando a morte adipocitária por apoptose (Borges \& Scorza, 2016). Além da hiperplasia adipocitária, a técnica da criolipólise também pode provocar queimaduras. Essa intercorrência não é comum, e geralmente acontece quando profissionais cometem erros técnicos por não serem capacitados para executar a técnica; manuseiam o aparelho de forma indevida ou pelo uso de membranas falsificadas ou reaproveitadas (Aguiar, 2020). Quando os profissionais entrevistados foram questionados sobre intercorrências com a criolipólise, nenhum referiu ter acontecido casos de hiperplasia adipocitária, nem queimaduras. A única intercorrência citada foi a presença de hematomas em apenas 3,6\% dos entrevistados.

Hematomas também foram intercorrências referidas por 3,6\% dos participantes quando se referiram a técnica de microagulhamento. Além dos hematomas também houve relatos $(3,6 \%)$ de manchas no local da aplicação da técnica. O microagulhamento provoca lesões através de microperfurações (Borges \& Scorza, 2016). Dessa forma, de acordo com Albano (2018) apud Negrão (2015) o microagulhamento é um procedimento que também apresenta riscos de causar complicações. Geralmente, as intercorrências podem acontecer por diversos fatores, como não escolher apropriadamente o equipamento, executar a técnica de forma inadequada, utilizar substâncias com potencial alergênico, má associação terapêutica, entre outros. 
De acordo com Milani (2020), a carboxiterapia é uma técnica onde ocorre infusão do gás carbônico medicinal, provocando um deslocamento do tecido. Pode ocorrer sensação de ardência durante a aplicação, dormência ou pequenos hematomas ao fim da aplicação (Felizzola \& Mejia, 2014 apud Anjos, 2019). Os estudos comprovam que a carboxiterapia se mostra um método seguro e eficaz em vários tratamentos como celulite, estrias e gordura localizada. No entanto, 14,3\% relataram ter intercorrências com a técnica, sendo 10,7\% hematomas e 3,6\% entre hiperemia acentuada, edema e hiperplasia adiposa paradoxal. O relato da hiperplasia adipocitária foi uma curiosidade para as pesquisadoras, uma vez que só foram encontrados registros dessa intercorrência nos estudos que se referiam a criolipólise. Ainda assim, a literatura aponta que a hiperplasia adipocitária paradoxal é uma intercorrência rara e mais presente nos homens que se submeteram a técnica da criolipólise (Borges \& Scorza, 2016).

De acordo Borges (2020), a microgalvanopuntura é uma técnica que associa os efeitos da corrente contínua junto com estímulo físico da agulha, gerando lesões traumáticas, vasodilatação e leve edema. Apenas 3,6\% dos profissionais relataram que a técnica de microgalvanopuntura causou hematomas.

Borges e Scorza (2016) afirmam que a endermologia é capaz de incrementar a circulação sanguínea e o fluxo linfático através da mobilização tecidual promovida pela sucção do tecido (pressão negativa). Este procedimento constitui de uma "prega móvel" na pele, com tamanhos variados de acordo com a regulagem da pressão negativa do vácuo, produzindo-se assim uma mobilização profunda na pele e tecido subcutâneo (Filippo \& Salomão, 2012 apud Gouveia, et al., 2018). 25\% dos profissionais relataram ter intercorrências com uso da endermologia, dentre estes 14,3\% petéquias e as demais intercorrências estão hematomas, manchas no local e hiperemia acentuada.

Silva, et al (2019) afirma que, entre os diversos efeitos fisiológicos da terapia com ondas de choque, se destaca o mecanismo que induz a liberação de fatores de crescimento e aumento da circulação do local. Com o equipamento de ondas de choque, 3,6\% dos entrevistados relataram que houve hiperemia acentuada e queimaduras como intercorrências.

De acordo Daniel e Moura (2018), a terapia combinada associa o uso de ultrassom de alta potência com estímulos musculares. Wanderley e Araújo (2019), traz que o ultrassom e a estimulação elétrica são modalidades terapêuticas que quando usadas simultaneamente se torna uma terapia combinada. Cerca de $10,7 \%$ dos profissionais relataram que tiveram intercorrências como hematomas e queimaduras com uso dessa associação.

Todos esses dados são demonstrados, conforme a tabela a seguir: . 
Tabela 2. Questionário sobre intercorrências em tratamentos estéticos corporais. Vitória da Conquista. 2021.

\begin{tabular}{|c|c|}
\hline Teve algum tratamento corporal que causou intercorrência & \\
\hline Não & $89,3 \%$ \\
\hline Sim & $10,7 \%$ \\
\hline \multicolumn{2}{|l|}{ Intercorrências com Ultrassom } \\
\hline Não & $100 \%$ \\
\hline \multicolumn{2}{|l|}{ Intercorrências com Radiofrequência } \\
\hline Não & $\mathbf{9 2 , 9 \%}$ \\
\hline Sim & $7,1 \%$ \\
\hline Petequias; & $3,6 \%$ \\
\hline Queimaduras & $3,6 \%$ \\
\hline \multicolumn{2}{|l|}{ Intercorrências com Criolipólise } \\
\hline Não & $96,4 \%$ \\
\hline Sim & $3,6 \%$ \\
\hline Hematomas & $3,6 \%$ \\
\hline \multicolumn{2}{|l|}{ Intercorrências com Microagulhamento } \\
\hline Não & $96,4 \%$ \\
\hline Sim & $3,6 \%$ \\
\hline Hematomas, manchas no local & $3,6 \%$ \\
\hline \multicolumn{2}{|l|}{ Intercorrências com Carboxiterapia } \\
\hline Não & $\mathbf{8 5 , 7 \%}$ \\
\hline Sim & $14,3 \%$ \\
\hline Hematomas & $10,7 \%$ \\
\hline Hiperemia acentuada; Hiperplasia adiposa paradoxal;Edema & $3,6 \%$ \\
\hline \multicolumn{2}{|l|}{ Intercorrências com Microgalvanopuntura } \\
\hline Não & $96,4 \%$ \\
\hline Sim & $3,6 \%$ \\
\hline Hematomas & $3,6 \%$ \\
\hline \multicolumn{2}{|l|}{ Intercorrências com Endermologia } \\
\hline Não & $75 \%$ \\
\hline Sim & $25 \%$ \\
\hline Petéquias & $14,3 \%$ \\
\hline Hematomas & $3,6 \%$ \\
\hline Hiperemia acentuada & $3,6 \%$ \\
\hline Manchas no local & $3,6 \%$ \\
\hline \multicolumn{2}{|l|}{ Intercorrências com Ondas de choque } \\
\hline Não & $96,4 \%$ \\
\hline Sim & $3,6 \%$ \\
\hline Hiperemia acentuada; Queimaduras & $3,6 \%$ \\
\hline \multicolumn{2}{|l|}{ Intercorrências com Terapia Combinada } \\
\hline Não & $89,3 \%$ \\
\hline Sim & $10,7 \%$ \\
\hline Hematomas; Queimaduras & $3,6 \%$ \\
\hline \multicolumn{2}{|c|}{ Utilizou alguma conduta para solucionar/ amenizar as intercorrências? } \\
\hline Não precisou utilizar nenhuma conduta & $\mathbf{7 1 , 4 \%}$ \\
\hline Utilizou outros recursos & $25 \%$ \\
\hline Encaminhou para tratamento médico & $3,6 \%$ \\
\hline
\end{tabular}

Fonte: Autores (2021).

Como registrado ao final da tabela acima, os profissionais foram questionados sobre as condutas utilizadas para solucionar/ amenizar a intercorrência, 71,4\% relataram que não precisou utilizar nenhuma conduta, 25\% utilizaram outros recursos e apenas $3,6 \%$ precisaram de encaminhamento médico.

O ideal é que sejam realizadas técnicas sem causar nenhuma intercorrência aos pacientes. Entretanto, sabe-se que elas podem acontecer e que podem ser mais simples, como hematomas, edemas, que podem regredir espontaneamente sem 
necessitar realizar nenhuma outra conduta; ou mais complicadas gerando consequências estéticas importantes que precisam de tratamento como manchas, queimaduras, hiperplasia adipocitária paradoxal. Por isso, o ideal é que o profissional tenha conhecimento e segurança para executar a técnica, e antes de realizá-la deve ser entregue um Termo de Consentimento Livre e Esclarecido (TCLE) aos seus pacientes informando sobre a técnica que será utilizada, os cuidados que devem ter após o procedimento e as possíveis intercorrências que o recurso pode causar. Mesmo com o TCLE, caso aconteça alguma intercorrência fica sendo responsabilidade do profissional em resolver as intercorrências ou direcionar para que outro profissional resolva.

\section{Conclusão}

Por meio deste estudo, foi possível analisar os relatos sobre as intercorrências em tratamentos estéticos corporais que vão desde as mais simples como, edemas e hematomas, quanto as mais complexas que são queimaduras, manchas e hiperplasia adiposa paradoxal. Os recursos terapêuticos que tiveram maiores relatos de intercorrências foram a endermologia (25\%), a carboxiterapia (14,3\%), a terapia combinada $(10,7 \%)$ e a radiofrequência $(7,1 \%)$. A maioria dos participantes que relataram ter passado por intercorrências não precisaram tomar outras medidas, pois as intercorrências foram simples, e apenas 3,6 \% deles referiram precisar encaminhar seus pacientes para atendimento médico. Entretanto, quando ocorreram intercorrências mais complexas, os profissionais precisaram tratar ou encaminhar seus pacientes para atendimento médico.

O presente estudo se torna relevante para que profissionais da área se atentem as possibilidades de intercorrências em diferentes recursos terapêuticos e assim, possam ter mais cuidado para conduzir seus atendimentos com maior segurança. Os desafios para elaboração deste estudo foi encontrar pesquisas atuais que abordassem sobre intercorrências em tratamentos estéticos. Por isso, sugerimos que novos estudos com essa temática sejam realizados, pois, se trata de um tema muito importante na atualidade. O conhecimento sobre as intercorrências nas abordagens terapêuticas é de grande importância para que os atendimentos ocorram da maneira mais segura, minimizando a exposição dos pacientes a riscos.

\section{Referências}

Aguiar, A. C. C. (2020) Eficáciada criolipólise na redução de lipodistrofia localizada e seus aspectos fisiológicos. Trabalho de conclusão de curso (Bacharelado em Biomedicina) - UniCEUB. Brasília.

Anjos, N. R. dos. (2019) Carboxiterapia no tratamento de estrias. Monografia (Bacharelado em Fisioterapia) - a Faculdade de Educação e Meio Ambiente FAEMA. Ariquemes - RO.

Albano, R. P. S. (2018) microagulhamento - a terapia que induz a produção de colágeno - revisão de literatura. Revista Saúde em Foco. (10), $456-473$.

Bastos, G. R., \& Nogueira, A. P. S. (2020) Os Benefícios da Carboxiterapia no Tratamento da Adiposidade Abdominal: Uma Revisão Integrativa. ID online revista de psicologia, 14(51), 157-167.

Borges, F. S., \& Scorza, F. A. (2016) Terapêutica em estética: conceitos e técnicas. Phorte.

Borges, F. S. T (2020). Efeitos da microgalvanopuntura e da puntura seca em estrias albas - estudo randomizado, controlado.Dissertação (Mestrado em Fisioterapia Dermatofuncional) - Escola Superior de Saúde do Instituto Politécnico do Porto. Portugal.

Brito, R. S. Santos, H. O. dos., Nascimento, R. S., \& Lima, M. V. A. (2019) Associação de protocolos em eletroterapia na redução de tecido adiposo subcutâneo. Brazilian Journa lof Health Review, 2(4), 3634-3650.

Daniel, B. F. R., \& Moura, T. S. (2018) O efeito da terapia combinada: Ultrassom e corrente aussie para adiposidade abdominal. Uniersitário Revista cientifica do unisalesiano, (18), 656-862.

Feller, A. G., Silva, E.da., \&Zimmermann, C. E. P. (2018) Utilização do ultrassom estético na gordura localizada. Revista saúde integrada, 11(21), 69-81.

Filgueiras, N. L. (2018) O crescimento e valorização do mercado de estética no Brasil. Trabalho de conclusão de curso (graduação em Arquitetura e Urbanismo). Faculdade de Ciências Gerenciais de Manhuaçu. Manhuaçu/MG.

Gouveia, L., Nunes, G., Pereira, L., \& Assis, I. (2018) Atuação da endermoterapia/vacuoterapia no tratamento do fibro edema gelóide - revisão de literatura. Revista Saúde em Foco - n. 10.

Milani, C. C. (2020) Efeitos da carboxiterapia como tratamento estético. Revista Extensão, 4(1). 
Research, Society and Development, v. 10, n. 16, e133101623507, 2021

(CC BY 4.0) | ISSN 2525-3409 | DOI: http://dx.doi.org/10.33448/rsd-v10i16.23507

Oliveira, A. L., Perez, E., Souza, J. B., \& Vasconcelos, M. G (2014) Curso didático de estética. (2a ed.), Yendis.

Pereira, R. R. (2019) Tecnologias no manejo do tecido adiposo. Trabalho de conclusão de curso (Graduação em Biomedicina) - Universidade de Cuiabá. Sinop/MT.

Queiroz, S. K. D., Rodrigues, G. S. C., \& Conti, M. H. S. (2021) Técnica de Microagulhamento no tratamento de estrias: uma revisão de literatura. Brazilian Journal of Development, 7(1), 4497-4519.

Silva, G.A., Costa, L. L., \& Caron, C. V. (2017) Aplicação da radiofrequênciana adiposidade localizada no abdômen. Trabalho de conclusão de curso (Tecnólogaem Cosmetologia Estética) - Universidade do sul de Santa Catarina.

Silva, R. M. V., Borges, F. S., Silva, J. D. C., Lira, J. C. S. O., Oliveira, M. P. M., Bezerra, L. C. B., Bezerra, B. N., \& Meyer, P. F. (2019) Efeitos das ondas de choque piezoelétrica na adiposidade localizada. ConScientiae Saúde, 18(1), 74-84.

Tassinary, J., Sinigaglia, M., \& Sinigaglia, G. (2018) Raciocínio clínico aplicado a estética corporal. Lajeado:Estética Experts.

Trindade, A. P., Amorim, M. T., Ferreira, J. A., Lima, C. M. V., Amaro, B. O., Ferreira, J. F. L., Holanda, G. H., Silveira, M. A., Santos, G. F. S., \& Baltazar, C. S. (2020) Perfil do biomédico esteta e a segurança do paciente em procedimentos estéticos: uma revisão integrativa. Revista Eletrônica Acervo Saúde, 12(10), e4783.

Wanderley, C. B., \&Araújo, I.W. (2019) Terapia combinada no tratamento da adiposidade abdominal: ensaio clínico randomizado. Projeto de pesquisa de iniciação cientifica do curso (Graduação fisioterapia) - Centro Universitário CESMAC. Maceió- AL. 